\title{
EROSIÓN HÍDRICA Y SU RELACIÓN CON EL AVANCE DE LA AGRICULTURA EN EL SUDESTE BONAERENSE, ARGENTINA. EL CASO DEL PARTIDO DE BENITO JUÁREZ DURANTE LAS CAMPAÑAS 1989/90, 2002/03 Y 2014/15
}

\author{
Nahuel David Sequeira ${ }^{1}$, Patricia Vazquez ${ }^{2}$, Mónica Sacido ${ }^{3}$
}

Universidad Nacional del Centro y Universidad Nacional de Rosario

\begin{abstract}
RESUMEN
La erosión hídrica es uno de los fenómenos más significativos de degradación del suelo a nivel mundial. Este trabajo estima la tasa de erosión hídrica actual y potencial y la variación de la pérdida total de suelos agrícolas de las unidades ecológicas del partido de Benito Juárez, para las campañas 1989/90, 2002/03 y 2014/15, estableciendo una relación entre estos valores y el avance agrícola. Metodológicamente, se superpusieron capas de información de trabajos antecedentes, para analizar la transformación de la superficie agrícola en cada unidad ecológica. Luego, se estimaron las tasas de erosión y la pérdida total de suelos; analizando su variación en el mismo lapso temporal y comparando estos valores con los niveles de tolerancia de pérdida de los suelos de la región. Para las 3 campañas, las mayores tasas de erosión corresponden a la unidad "Sierras"; y las mayores pérdidas de suelos a la unidad "Llanura ondulada, suavemente ondulada y deprimida". Se plantea que existe una relación directa entre los resultados obtenidos y el incremento de la superficie destinada al avance de la agricultura. Finalmente, los datos alcanzados contribuyen al diagnóstico acerca de la sostenibilidad del modelo productivo del Partido, en pos de un correcto ordenamiento ambiental del territorio.

Palabras clave: clasificaciones supervisadas; unidades ecológicas; indicador de erosión; teledetección y sistemas de información geográfica; ordenamiento ambiental del territorio.

\section{WATER EROSION AND ITS RELATIONSHIP WITH THE ADVANCE OF AGRICULTURE IN THE 1989/90, 2002/03 AND 2014/15 CAMPAIGNS} SOUTHEASTERN BUENOS AIRES, ARGENTINA. THE CASE OF BENITO JUAREZ DURING

\section{ABSTRACT}

Water erosion is one of the most significant soil degradation phenomena worldwide. This work estimates the current and potential rate of water erosion and the variation of the total loss of agricultural soils of the ecological units of the Benito Juárez county, for the 1989/90, 2002/03 and 2014/15 campaigns, establishing a relationship between these values and the agricultural advance. Methodologically, layers of previous information were superimposed to analyze the transformation of the agricultural area in each ecological unit. Then, the rates of erosion and the total loss of soils were estimated; analyzing their variation in the same time lapse and comparing these values with the tolerance levels of loss of soils in the region. For the 3 campaigns, the highest erosion rates correspond to the "Mountain ranges" unit; and the highest soil losses to the "Wavy, gently wavy and depressed plains" unit. It is proposed that there is a direct relationship between the results obtained and the increase in the area destined to the advance of agriculture. Finally, the data reached contribute to the diagnosis about the sustainability of the productive model of the County, in pursuit of a correct environmental territorial ordering.

Key words: supervised classifications; ecological units; erosion indicator; remote sensing and geographic information systems; environmental territorial ordering
\end{abstract}

\footnotetext{
${ }^{1}$ Centro de Estudios Sociales de América Latina (CESAL), Facultad de Ciencias Humanas, Universidad Nacional del Centro de la provincia de Buenos Aires. CONICET. E-mail: nahuelsequeira@ conicet.gov.ar ${ }^{2}$ CESAL, Facultad de Ciencias Humanas, Universidad Nacional del Centro de la provincia de Buenos Aires. CONICET. E-mail: patriciavazquez@conicet.gov.ar

3 Cátedra de forrajes. Facultad de Ciencias Agrarias, Universidad Nacional de Rosario. E-mail: msacido@hotmail.com
}

Fecha de recepción: 11 de septiembre de 2019. Fecha de aceptación: 26 de diciembre de 2019 


\section{INTRODUCCIÓN}

El ordenamiento ambiental es entendido como aquel ordenamiento del territorio que introduce la dimensión ambiental en su conceptualización, diferenciándose del enfoque economicista que considera la ordenación territorial sin tener en cuenta el costo social y el impacto ambiental que ello signifique, ignorando la realidad social y el sentido de utilización de la naturaleza dentro de los criterios de uso sostenible. Diferenciándose, además, de la conceptualización proteccionista que plantea como finalidad exclusiva la protección del ambiente, olvidando el contexto social y económico de los recursos naturales, así como, la necesidad de su aprovechamiento para conseguir el bienestar común (MARTÍNEZ e IGLESIAS, 2006).

Dentro de estos recursos naturales, el suelo ocupa un rol de vital importancia, debido a sus múltiples funciones ambientales. Se considera no renovable comparado con la escala humana, desarrollándose lentamente y demostrando una gran variabilidad espacial (DÍAZ PEREIRA y ANAYA ROMERO, 2011).

Aquellos procesos que provocan una disminución de la capacidad productiva de los suelos, son denominados degradativos; y, entre ellos, la erosión (hídrica y eólica) resulta ser uno de los más relevantes (FAO, 1980). Si bien se trata de un proceso natural, debido al uso intensivo de las tierras agrícolas, al cambio de coberturas y al manejo inadecuado; la dinámica del paisaje se ha modificado, y los ritmos de afectación se están acelerando (FAO, 1990).

La erosión hídrica se ha convertido en uno de los fenómenos más significativos de degradación del suelo a nivel mundial, provocando serios impactos ecológicos y elevados costos económicos. A través de sus efectos sobre la producción agrícola, la infraestructura y la calidad del agua que, a su vez, afectan la calidad de la población, puede llegar a amenazar la seguridad alimentaria y representar un serio problema para el desarrollo sustentable (NÁJERA GONZÁLEZ et al., 2016).

Lo mencionado se manifiesta como uno de los principales problemas ambientales de las regiones en las que se llevan a cabo actividades agrícolas. En relación a ello, Ferreras et al. (2015) afirman que alrededor de una cuarta parte de las tierras cultivables del planeta presentan algún signo de degradación y/o erosión.

Para muchos países latinoamericanos, esta problemática se ha convertido en una de las que ocasionan impactos negativos de mayor gravedad, favoreciendo la generación de estados más pobres y con mayores riesgos ambientales (GVOZDENOVICH et al., 2017).

Considerando que, según sus potencialidades y limitaciones, el papel del suelo es crucial dentro del ordenamiento territorial de cualquier región; las transformaciones en su composición, calidad o disponibilidad deben ser monitoreadas, con la finalidad de identificar situaciones problemáticas y promover prácticas de manejo que favorezcan la preservación de los múltiples servicios que presta (DÍAZ PEREIRA y ANAYA ROMERO, 2011).

La organización de las actividades humanas, permite disminuir los efectos negativos sobre el suelo, beneficiando sobre todo a las comunidades agrícolas que hacen uso directo del recurso (FAO, 1980). En relación a ello, aunque además de adherir a diversos programas, convenciones y tratados, la Argentina llevó a cabo grandes esfuerzos a nivel local para accionar en contra de los procesos degradativos; no ha logrado impedir que gran parte de su territorio evidencie algún nivel de degradación (HERNÁNDEZ y DUFILHO, 2012).

Ante esta situación, y con la finalidad de intentar disminuirla, en el país comenzó a adoptarse a fines de los `80 la Siembra Directa (SD) o labranza cero (LÓPEZ et al., 2015), destacándose como uno de los medios más difundidos para el control de la erosión (ARES et al., 2014). Se trata de una práctica que consiste en mantener el rastrojo del cultivo anterior sobre la superficie, sin efectuar laboreos y combatir las malezas con herbicidas (GONZÁLEZ y ROMÁN, 2009); la cual, según estimaciones de Nocelli Pac (2018), para la campaña 2014/15 ya se implementaba en un 90\% de la superficie agrícola del país.

Sin embargo, Casas (2015) estimó que aproximadamente 64,6 millones de hectáreas del territorio nacional se encontraban afectadas por erosión hídrica, resultando este valor un $150 \%$ más que las 25 millones calculadas por FECIC-PROSA (1988) en estudios anteriores.

Considerando lo hasta aquí indicado, es posible observar que la Región Pampeana Argentina (RPArg) resultó una de las áreas mayormente afectadas. Cerdá et al. (2014) plantean que, durante 
mucho tiempo, el modelo de producción se vio representado por una alternancia entre ganadería y agricultura. No obstante, una supuesta mayor rentabilidad, en conjunto con la falta de comprensión de los servicios ecológicos que prestaba la ganadería pastoril, estimuló una marcada agriculturización en la región. Como resultado, comenzó a evidenciarse una rápida transformación del paisaje, donde los suelos de la RPArg sufrieron un acelerado agotamiento de nutrientes (MANCHADO, 2010), y se originaron una serie de profundas transformaciones dentro de los agroecosistemas. Entre ellas, puede destacarse a la erosión y la pérdida de fertilidad observadas en las principales cuencas productivas de la región como algunas de las más importantes consecuencias (ALTIERI, 1999; SANS, 2007; GARCÍA y SALVAGIOTTI, 2009).

Este proceso de agriculturización y sus derivaciones no se expresan uniformemente (GONZÁLEZ y ROMÁN, 2009; BILENCA et al., 2012); sino que sus consecuencias derivan, entre otros factores, del sistema de agricultura implementado, el tipo de tecnologías aplicadas y de las características ecológicas propias de cada área o paisaje.

En tal contexto, uno de los conceptos de mayor interés es el de definición de unidades de paisaje, denominadas en este trabajo como Unidades Ecológicas (UEcs). Mediante este proceso, a partir de la utilización de determinados enfoques de clasificación y la integración de atributos bióticos y abióticos, es posible definir y representar cartográficamente unidades que presentan un cierto grado de homogeneidad (SERRANO GINÉ, 2012; SALINAS CHÁVEZ y RAMÓN PUEBLA, 2013). Teniendo en cuenta sus aptitudes y características, cada unidad ecológica (UEc) presenta condicionantes (o no), ante las actividades que pueden ser desarrolladas en ella (ZULAICA et al., 2018).

El partido de Benito Juárez, dentro de la RPArg, se ha desarrollado históricamente como un área de explotación mixta, con preponderancia ganadera. Sin embargo, puede observarse que a través de los años no resultó ajeno al proceso de agriculturización (REQUESENS y SILVA, 2011) $\mathrm{y}$, consecuentemente, tampoco a sus efectos negativos (SEQUEIRA et al., 2015).

Pese a la gravedad de la situación, son escasos los informes que tratan acerca del avance de este proceso y su relación con la erosión y pérdida de suelos a escala de Partido; lo cual resultaría sumamente relevante para lograr el desarrollo de una efectiva organización en el manejo de la tierra y las actividades agrícolas desarrolladas en ella, tendientes a lograr un ordenamiento ambiental del territorio.

Por lo tanto, considerando la situación del Partido, que la erosión hídrica es uno de los principales problemas que comprometen la sustentabilidad de todos los sistemas productivos, y que la estimación de las pérdidas de suelo por este fenómeno es una información de gran importancia para la planificación y toma de decisiones a diferentes niveles (GAITÁN et al., 2017); el objetivo de este trabajo es estimar la tasa de erosión hídrica actual (A) y potencial (EP) y la variación de la pérdida total de los suelos agrícolas presentes en las UEcs del partido de Benito Juárez, para las campañas 1989/90, 2002/03 y 2014/15, estableciendo una relación entre las variaciones de estos valores y el avance de la agricultura.

\section{2. ÁREA DE ESTUDIO}

El partido de Benito Juárez se encuentra en el sudeste bonaerense (Figura 1), dentro de la RPArg. De los $5285 \mathrm{~km}^{2}$ que abarca el territorio, solamente un $19,15 \%$ demuestra capacidad agrícola o agrícola-ganadera, mientras que el resto cuenta con tierras con características apropiadas para uso ganadero $(44,76 \%)$ o ganadero-agrícola $(36,08 \%)$ (SOCIEDAD RURAL DE BENITO JUÁREZ, 2007). 


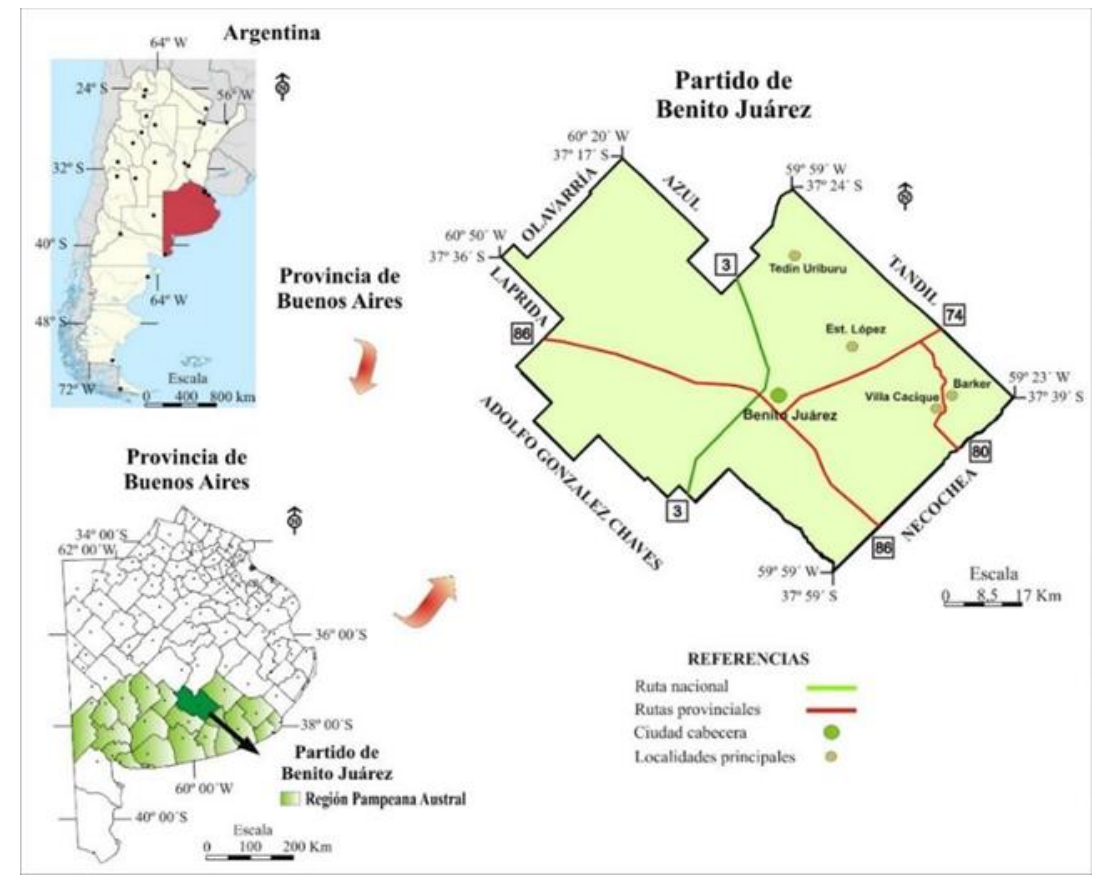

Fuente: Elaboración personal

En cuanto a la vegetación originaria de la región, la comunidad de mayor predominancia es la estepa de flechillas, de gran riqueza en gramíneas stipeas de los géneros Stipa y Piptochaetium, las cuales se combinan en diversas formas (CABRERA, 1976).

Debido a que los factores climáticos no son condicionantes del desarrollo agroproductivo, y que los suelos demuestran gran aptitud para este tipo de actividades, se ha producido una enorme sustitución de la vegetación originaria por agroecosistemas (VAZQUEZ et al., 2013).

Con relación al proceso de agriculturización evidenciado en los últimos años, según Sequeira et al. (2018) se observa que en el período 1990-2003 la superficie dedicada a agricultura se incrementó en un $111,24 \%$, en detrimento de la destinada a la actividad ganadera, la cual disminuyó en un $13,78 \%$. Por su parte, para el período 2003-2015, la tendencia se repitió con valores del 124,78 y $43,41 \%$, respectivamente.

Las áreas destinas a agricultura se encuentran distribuidas sobre ecosistemas que presentan características particulares, lo cual permite diferenciarlos. Considerando lo planteado por Sequeira et al. (2019), en el Partido pueden diferenciarse 5 UEcs con rasgos específicos.

Dentro de la UEc1 (Sierras), los subgrupos de suelos dominantes son Hapludoles líticos, Hapludoles típicos y Argiudoles típicos; y la textura superficial y subsuperficial es del tipo franca. La unidad muestra un predominio de cursos de agua con régimen permanente y presencia de afloramiento rocoso en cúspides y laderas, un drenaje de bien a algo excesivamente drenado, escurrimiento de medio a muy rápido y permeabilidad de moderadamente lenta a moderada. Los valores de las pendientes varían entre el 1 y $10 \%$.

En la UEc2 (Llanura periserrana y ondulada), los subgrupos de suelos de mayor importancia son Argiudoles típicos y Hapludoles petrocálcicos; la textura superficial es franco-limosa y la subsuperficial franco-arcillo-limosa. Existe un predominio de cursos de agua con régimen permanente y no permanente, las condiciones de drenaje son buenas, el escurrimiento de medio a rápido y la permeabilidad de moderadamente lenta a moderada. Los valores de pendientes rondan entre el 1 y $10 \%$,

Para la UEc3 (Llanura ondulada, suavemente ondulada y deprimida), los subgrupos de suelos dominantes son Argialboles típicos, Argiudoles típicos, Argiudoles ácuicos y Natracuoles típicos; la textura superficial varía desde franco a franco limosa y la subsuperficial desde franco arcillosa a franco arcillo limosa. Es posible observar escasos cursos de agua con régimen permanente y no permanente, y la presencia de bañados permanentes e importantes lagunas. Se presentan buenas condiciones de drenado, el escurrimiento es de medio a rápido y la permeabilidad moderada, y las pendientes varían entre 1 y $3 \%$. 
En relación a la UEc4 (Llanura deprimida), los subgrupos de suelos más relevantes son Argiudoles típicos, Argiudoles ácuicos, Natracuoles típicos, Natralboles típicos y Argialboles típicos; la textura superficial es franco-limosa y la subsuperficial franco-arcillo-limosa. Se manifiesta la presencia de bañados permanentes y no permanentes, lagunas y cursos con régimen permanente y no permanente. El drenaje varía desde pobremente drenado a bien drenado, el escurrimiento de muy lento a rápido y la permeabilidad de moderadamente lenta a muy lenta. Las pendientes varían entre el 0 y $3 \%$.

Por último, en la UEc5 (Llanura deprimida con cursos de agua y áreas inundables) los subgrupos de suelos dominantes son Argiudoles típicos, Argiudoles ácuicos, Argiacuoles típicos, Natracuoles típicos, Natracualfes típicos, Natralboles típicos y Hapludoles tapto nátricos. La textura superficial es franco-limosa y la subsuperficial varía desde arcillosa a arcillo-limosa y franco-arcillo-limosa. Es posible observar gran cantidad de cursos de agua con régimen permanente y no permanente, y la presencia de importantes lagunas y gran cantidad de bañados permanentes. Las condiciones de drenaje resultan pobres, el escurrimiento lento, la permeabilidad es de moderadamente lenta a lenta, y los valores de las pendientes oscilan entre el 0 y $1 \%$.

\section{MATERIALES Y MÉTODOS}

Para una mejor organización, este apartado fue dividido en dos etapas. En primer lugar, se identificó la transformación de la superficie agrícola en cada UEc del Partido para 1990, 2003 y $2015^{4}$, a partir de la superposición de capas de información obtenidas en trabajos antecedentes. Por otra parte, se efectuó una estimación de A y EP, y de la pérdida total de suelos; analizando su variación en el mismo lapso temporal y comparando estos valores con los niveles de tolerancia (Tol) de pérdida de los suelos de la región.

\subsection{PRIMERA ETAPA}

Con el propósito de obtener la superficie ocupada por la agricultura en cada UEc, se prosiguió a superponer capas de información obtenidas de trabajos previos, mediante la utilización del software ENVI (4.5).

En este sentido, se empleó el trabajo de Sequeira et al. (2018), donde se determinó el avance de la agricultura en el Partido a través de 3 clasificaciones supervisadas (CS), efectuadas sobre imágenes satelitales Landsat 5 (TM) y 8 (OLI) concernientes a los años 1990, 2003 y 2015 (software ENVI 4.5). Por otra parte, se analizó el trabajo de Sequeira et al. (2019) en el cual, a partir de la integración de atributos geomorfológicos, edáficos (series de suelo), hidrológicos y de vegetación, se definieron y caracterizaron las diversas UEcs que componen el Partido; arrojando como resultado unidades de carácter homogéneo y diferenciables entre sí.

A partir de la superposición de las CS y las UEcs se adquirieron los valores de superficie de cada clase de uso ${ }^{5}$ existente por UEc en cada año. Para ello, mediante el software ENVI (4.5), se crearon 5 máscaras (una por cada UEc) que fueron aplicadas de manera individual sobre las 3 CS; y, a través de la utilización de función Compute Stadistics, se obtuvieron los valores estadísticos correspondientes.

Finalmente, la información conseguida acerca del área destinada a la actividad agrícola en cada UEc del Partido, resulta clave para luego estimar la pérdida de suelo total a lo largo de los períodos evaluados.

\subsection{SEGUNDA ETAPA}

\footnotetext{
${ }^{4}$ El interés en analizar las fechas seleccionadas reside en que, desde la década del 90, dentro del Partido comenzó a sucederse un importante desarrollo de la agricultura, aumentando las tierras utilizadas para cultivos a expensas de aquellas empleadas para ganadería. Además, a partir del año 2002 comienza a verificarse con mayor claridad el proceso de agriculturización en la región. Finalmente, los datos acerca del año 2015 se encuentran dentro de los más actualizados y de mayor fiabilidad.

${ }^{5}$ En este trabajo únicamente fueron considerados aquellos concernientes a la actividad agrícola.
} 
A partir de las UEcs mencionadas anteriormente, se identificaron las series de suelos dominantes en cada una y su proporción en relación al total de la superficie, con el fin de estimar A y EP.

La estimación de la tasa de erosión propiamente dicha, se realizó a través de la Ecuación Universal de Predicción de Erosión Hídrica (USLE) establecida por Wischmeier y Smith (1978), la cual ha sido utilizada ampliamente alrededor del mundo (BONILLA et al., 2010; NÁJERA GONZÁLEZ et al., 2016), debido a su capacidad de aplicación a distintas áreas, bajo costo cuando existen datos de base disponibles y su eficacia para identificar regiones susceptibles a la erosión (HERNÁNDEZ y DUFILHO, 2012):

$$
\mathrm{A}=\mathrm{R} * \mathrm{~K} * \mathrm{~L} * \mathrm{~S} * \mathrm{C} * \mathrm{P}
$$

donde A: tasa de erosión actual (ton ha ${ }^{-1}$ año $\left.^{-1}\right)$; R: Erosividad de las lluvias ( $\mathrm{hJ} \mathrm{cm} \mathrm{m}^{-2} \mathrm{~h}^{-1}$ ); $\mathrm{K}$ : Susceptibilidad del suelo a la erosión hídrica (ton $\mathrm{m}^{2} \mathrm{~h} \mathrm{ha}^{-1} \mathrm{hJ}^{-1} \mathrm{~cm}^{-1}$ ); L: Longitud de la pendiente (m); S: Pendiente (\%); C: Factor cultivo o cobertura (adimensional) y P: Factor práctica conservacionista (adimensional).

El factor R, que representa valores de lluvia y escurrimiento (GAITÁN et al., 2017), se obtuvo considerando los dominios edáficos que caracterizan el área (IRURTIA y CRUZATE, 2002).

En cuanto a la estimación del factor $\mathrm{K}$, fue necesaria información edáfica específica de los perfiles de suelos, tal es el caso del \% de materia orgánica, \% de limo y arena muy fina, grado de estructura, permeabilidad y \% de arcilla (GAITÁN et al., 2017).

Los factores L y S son analizados en conjunto y se los denomina factor topográfico (LS), el cual establece el aporte que hace el relieve a la erosión hídrica. Los datos de longitud y gradiente fueron adquiridos de información proveniente de Irurtia y Cruzate (2002) y la consulta en línea de las cartas de suelos (escala 1:50.000) de INTA (Instituto Nacional de Tecnología Agropecuaria), respectivamente. Por último, tanto el factor $\mathrm{C}$ como el $\mathrm{P}$ dependen directamente del tipo de manejo que cada productor lleve a cabo dentro de su establecimiento.

El producto final resultante de estos seis factores, permite estimar A para una situación determinada de clima, relieve, suelo, cultivo y manejo. Por su parte, el valor de EP se obtiene a partir del producto de los factores R, K y LS; y es definida como la máxima posible para un sitio determinado, considerando un suelo desnudo durante todo el año. Con el objetivo de estimar la erosión mediante la mencionada ecuación, se utilizó la aplicación web desarrollada por Gvozdenovich et al. (2015).

Los resultados de A fueron comparados con los valores de Tol estimados por INTA (GAITÁN et al., 2017), la cual hace referencia a la tasa máxima de erosión que permite mantener un alto nivel de productividad. En ese informe, los autores establecieron niveles de Tol variables de acuerdo al régimen climático y la profundidad del suelo; correspondiendo las mayores tasas a suelos profundos de zonas húmedas (mayor tasa de formación de suelo) y las más bajas a suelos someros de zonas áridas (baja tasa de formación de suelo).

Para establecer esta comparación, se analizó la profundidad del suelo de cada una de las series dominantes mencionadas, y las condiciones climáticas de la región donde se localizan. En base a ello, se estableció un límite de Tol para cada serie de suelo y, posteriormente, se realizó un promedio de estos datos obteniendo el valor del límite de Tol por UEc.

Finalmente, la información adquirida acerca de A y EP junto a la superficie destinada a agricultura en cada UEc, se utilizó para determinar la pérdida total de suelos por unidad, y su variación en los años analizados; obteniéndose información que será de gran utilidad al momento de plantear estrategias tendientes a una correcta ordenación ambiental del territorio.

\section{RESULTADOS Y DISCUSIÓN}

\subsection{SUPERPOSICIÓN DE CLASIFICACIONES SUPERVISADAS Y UNIDADES ECOLÓGICAS}


A continuación, en la Tabla 1 y Figura 2, pueden observarse los resultados obtenidos en relación a la superficie destinada a agricultura en cada UEc por año evaluado; los cuales fueron alcanzados luego de la superposición de las 3 CS del Partido (años 1990, 2003 y 2015) y de las 5 UEcs que lo componen.

TABLA 1

Superficie destinada a la actividad agrícola por UEc

\begin{tabular}{ccccccc}
\hline \multirow{2}{*}{ Año } & UEc1 & UEc2 & UEc3 & UEc4 & UEc5 & Total \\
\cline { 2 - 6 } & \multicolumn{5}{c}{ Superficie destinada a agricultura $\left(\mathrm{km}^{2}\right)$} \\
\hline $\mathbf{1 9 9 0}$ & 13,7 & 120,18 & 228,11 & 52,06 & 205,78 & 619,83 \\
\hline $\mathbf{2 0 0 3}$ & 24,48 & 234,24 & 478,04 & 72,01 & 500,57 & 1309,34 \\
\hline $\mathbf{2 0 1 5}$ & 30,38 & 422,33 & 1138,4 & 182,85 & 1169,2 & 2943,16 \\
\hline
\end{tabular}

Fuente: Elaboración personal

FIGURA 2

Superposición de UEcs y CS del partido de Benito Juárez
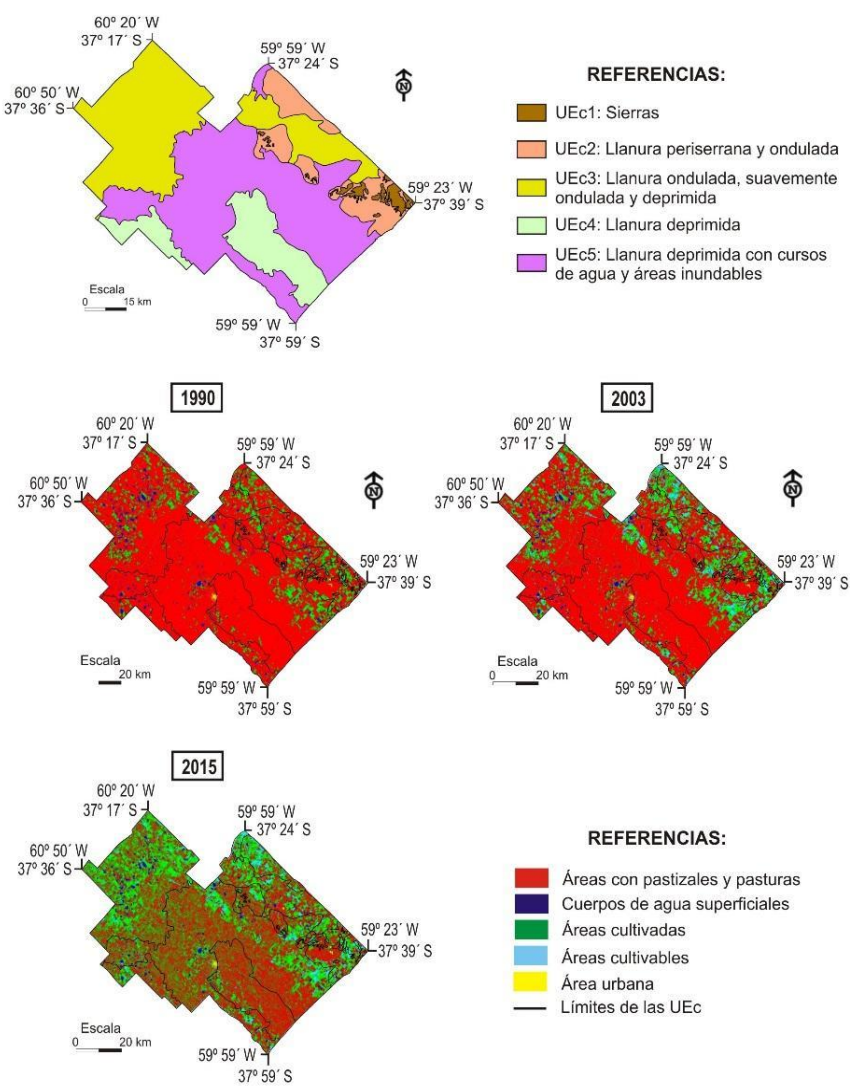

Fuente: Elaboración personal sobre la base de Sequeira et al. $(2018 ; 2019)$

\subsection{ESTIMACIÓN DE LA TASA DE EROSIÓN PARA LAS CAMPAÑAS 1989/90, 2002/03 Y 2014/15}

En base a sus características, los distintos suelos que componen las UEcs demuestran diversos grados de susceptibilidad a la erosión hídrica. Además, esta situación puede resultar disminuida o acentuada según las actividades desarrolladas en el territorio y las formas o prácticas de manejo llevadas a cabo.

Al momento de realizar la estimación de A y EP, fue preciso contar con datos sobre cada uno de los factores que componen la ecuación. Partiendo desde allí, la tasa de erosión fue calculada 
para cada UEc, considerando las series de suelos predominantes en cada caso y la superficie que ocupan cada una de ellas respecto al total de cada UEc.

Para el presente trabajo, el factor $\mathrm{R}$ mostró diferentes valores, los cuales dependieron de cada una de las unidades evaluadas. En la UEc1, representada por el dominio 1, el valor de $\mathrm{R}$ asciende a $306 \mathrm{hJ} \mathrm{cm} \mathrm{m}{ }^{-2}$. Por su parte, tanto la UEc2 como la UEc3 están caracterizadas por el dominio 2, con un valor de $\mathrm{R}$ igual a $312 \mathrm{hJ} \mathrm{cm} \mathrm{m}^{-2}$. Por último, la UEc4 y la UEc5 se encuentran representadas por el dominio 13 , con un valor de $\mathrm{R}$ igual a $318 \mathrm{hJ} \mathrm{cm} \mathrm{m}$.

En relación al factor $\mathrm{K}$, una vez aplicada la ecuación correspondiente, fue obtenido para cada una de las series de suelos que integran las UEcs (Tabla 2).

TABLA 2

UEcs: susceptibilidad de los suelos a erosión hídrica (factor K)

\begin{tabular}{|c|c|c|c|c|c|c|c|}
\hline Unidad & Serie & $\begin{array}{l}\text { MO } \\
(\%)\end{array}$ & $\begin{array}{l}\text { Limo + arena } \\
\text { muy fina }(\%)\end{array}$ & $\begin{array}{c}\text { Grado de } \\
\text { Estructura }\end{array}$ & $\begin{array}{c}\text { Permeabilidad } \\
\left(\mathrm{cm} \mathrm{hora}^{-1}\right)\end{array}$ & $\begin{array}{c}\text { Arcilla } \\
(\%)\end{array}$ & $\begin{array}{c}K \\
\left(h J \mathrm{~cm} \mathrm{~m}^{-2} h^{-1}\right)\end{array}$ \\
\hline \multirow{2}{*}{ UEc1 } & SP & 10 & 58,5 & 2 (granular fina) & $\begin{array}{l}4 \text { (moderadamente } \\
\text { lenta: } 0,5 \text { a } 2 \text { ) }\end{array}$ & 32,8 & 0,100 \\
\hline & $\mathrm{Az}$ & 7,69 & 68,2 & 3 (granular gruesa) & $\begin{array}{l}3 \text { (moderada: } 2 \mathrm{a} \\
6,25)\end{array}$ & 30,3 & 0,224 \\
\hline \multirow{3}{*}{ UEc2 } & $\mathrm{Ta}$ & 6,74 & 70,0 & 3 (granular gruesa) & 3 (moderada: $2 \mathrm{a}$ & 29,4 & 0,274 \\
\hline & $\mathrm{Az}$ & 7,69 & 68,2 & 3 (granular gruesa) & 3 (moderada: 2 a & 30,3 & 0,224 \\
\hline & $\mathrm{CC}$ & 6,02 & 30,9 & 3 (granular gruesa) & $\begin{array}{l}4 \text { (moderadamente } \\
\text { lenta: } 0,5 \text { a } 2)\end{array}$ & 30,7 & 0,176 \\
\hline \multirow{3}{*}{ UEc3 } & Ola & 7,27 & 61,9 & $\begin{array}{c}4 \text { (bloques } \\
\text { subangulares } \\
\text { medios) }\end{array}$ & 3 (moderada: 2 a & 33,8 & 0,252 \\
\hline & LLo & 3,78 & 32,7 & $\begin{array}{l}4 \text { (bloques } \\
\text { subangulares } \\
\text { medios) }\end{array}$ & $\begin{array}{c}3 \text { (moderada: } 2 \text { a } \\
6,25)\end{array}$ & 25,0 & 0,247 \\
\hline & $\mathrm{Az}$ & 7,69 & 68,2 & 3 (granular gruesa) & $\begin{array}{c}3 \text { (moderada: } 2 \text { a } \\
6,25)\end{array}$ & 30,3 & 0,224 \\
\hline \multirow{3}{*}{ UEc4 } & TA & 3,87 & 26,9 & 3 (granular gruesa) & $\begin{array}{l}4 \text { (moderadamente } \\
\text { lenta: } 0,5 \text { a } 2 \text { ) }\end{array}$ & 25,9 & 0,202 \\
\hline & LHe & 1,04 & 42,5 & $\begin{array}{c}4 \text { (bloques } \\
\text { subangulares } \\
\text { medios) }\end{array}$ & $\begin{array}{c}5 \text { (lenta: } 0,125 \text { a } \\
0,500)\end{array}$ & 14,8 & 0,488 \\
\hline & SIs & 1,36 & 34,5 & $\begin{array}{c}4 \text { (bloques } \\
\text { angulares medios) }\end{array}$ & $\begin{array}{c}6 \text { (muy lenta: } \\
\text { inferior a } 0,125 \text { ) }\end{array}$ & 32,4 & 0,380 \\
\hline \multirow{4}{*}{ UEc5 } & Cru & 1,72 & 46,7 & $\begin{array}{l}4 \text { (bloques } \\
\text { subangulares } \\
\text { medios) }\end{array}$ & $\begin{array}{l}4 \text { (moderadamente } \\
\text { lenta: } 0,5 \text { a } 2 \text { ) }\end{array}$ & 15,8 & 0,466 \\
\hline & Ele & 1,21 & 49 & 4 (laminar) & $\begin{array}{c}5 \text { (lenta: } 0,125 \text { a } \\
0,500)\end{array}$ & 13,9 & 0,546 \\
\hline & $\mathrm{CBu}$ & 1,60 & 45,2 & $\begin{array}{l}4 \text { (bloques } \\
\text { subangulares } \\
\text { medios) }\end{array}$ & $\begin{array}{c}5 \text { (lenta: } 0,125 \text { a } \\
0,500)\end{array}$ & 17,0 & 0,484 \\
\hline & LHe & 1,04 & 42,5 & $\begin{array}{c}4 \text { (bloques } \\
\text { subangulares } \\
\text { medios) }\end{array}$ & $\begin{array}{c}5 \text { (lenta: } 0,125 \text { a } \\
0,500)\end{array}$ & 14,8 & 0,488 \\
\hline
\end{tabular}

Fuente: Elaboración personal a partir de las ecuaciones planteadas por Wischmeier y Smith (1978) y la aplicación web de Gvozdenovich et al. (2015).

Por otra parte, la información necesaria para la obtención del factor LS se estimó en función de los suelos dominantes en cada UEc (Tabla 3). 
UEcs: Longitud de las pendientes y gradiente (factor LS)

\begin{tabular}{ccccc}
\hline Unidad & Serie & $\begin{array}{c}\text { Longitud de la } \\
\text { pendiente }(\mathbf{m})\end{array}$ & $\begin{array}{c}\text { Pendiente } \\
(\boldsymbol{\%})\end{array}$ & LS \\
\hline \multirow{2}{*}{ UEc1 } & $\mathrm{SP}$ & 300 & 6,5 & 2,36 \\
\cline { 2 - 5 } & $\mathrm{Az}$ & 300 & 2,0 & 0,47 \\
\hline \multirow{2}{*}{ UEc2 } & $\mathrm{Ta}$ & 300 & 2,0 & 0,47 \\
\cline { 2 - 5 } & $\mathrm{Az}$ & 300 & 2,0 & 0,47 \\
\cline { 2 - 5 } & $\mathrm{CC}$ & 300 & 5,5 & 1,86 \\
\hline \multirow{3}{*}{ UEc3 } & $\mathrm{Ola}$ & 300 & 2,0 & 0,47 \\
\cline { 2 - 5 } & $\mathrm{LLo}$ & 300 & 2,0 & 0,47 \\
\cline { 2 - 5 } & $\mathrm{Az}$ & 300 & 2,0 & 0,47 \\
\hline \multirow{3}{*}{ UEc4 } & $\mathrm{TA}$ & 200 & 2,0 & 0,42 \\
\cline { 2 - 5 } & $\mathrm{LHe}$ & 200 & 0,5 & 0,1 \\
\cline { 2 - 5 } & $\mathrm{SIs}$ & 200 & 0,25 & 0,06 \\
\hline & $\mathrm{Cru}$ & 200 & 0,5 & 0,1 \\
\cline { 2 - 5 } & $\mathrm{Ele}$ & 200 & 0,5 & 0,1 \\
\cline { 2 - 5 } & $\mathrm{CBu}$ & 200 & 0,5 & 0,1 \\
\cline { 2 - 5 } & $\mathrm{LHe}$ & 200 & 19 \\
\hline
\end{tabular}

Fuente: Elaboración personal a partir de las ecuaciones planteadas por Wischmeier y Smith (1978) y la aplicación web de Gvozdenovich et al. (2015).

El valor alcanzado para el factor $\mathrm{C}$, fue distinto según el momento considerado. La principal diferencia al calcularlo se debió a que, en la campaña 1989/90, la agricultura dentro del Partido era desarrollada mediante Siembra Convencional (SC) o tradicional. En contraposición, tanto para las campañas 2002/2003 y 2014/2015, esta práctica fue reemplazada por la SD, casi en la totalidad de la superficie. Para la primera fecha, el valor de C fue de 0,20082 y surgió de un promedio entre los valores de C correspondientes a los planteos "Trigo en rotación", "Girasol en rotación", "Maíz en rotación" " "Soja en rotación". En cuanto a las dos restantes, se promediaron los valores de los planteos "Maíz en rotación" y "Trigo/soja de segunda", alcanzando el factor C un valor de $0,06965^{6}$. Finalmente, debido a que no fue registrada la aplicación de ningún tipo de prácticas conservacionistas, el valor $\mathrm{P}$ asciende a 1 en la totalidad de los casos evaluados.

El resultado logrado a partir del producto de los factores antes mencionados $(\mathrm{R} * \mathrm{~K} * \mathrm{LS} * \mathrm{C} * \mathrm{P})$ facilitó el cálculo de A para cada una de las series de suelos analizadas. Por otra parte, el valor de A de cada UEc se obtuvo a partir de la sumatoria de los valores de cada serie multiplicados por la proporción que ocupa en la unidad.

Del mismo modo planteado para la estimación de A, surge el valor de EP. No obstante, en este caso solo es efectuado el producto entre los factores R, K y LS, sin considerar C ni P.

A continuación, en las Tablas 4 y 5 se evidencian los valores alcanzados para A y EP en las unidades sobre las que son desarrolladas las actividades agrícolas del Partido.

TABLA 4

Tasas de erosión actual (A) y potencial (EP) para la campaña 1989/90

\begin{tabular}{cccccccccc}
\hline Unidad & Serie & $\begin{array}{c}\text { Proporción } \\
(\boldsymbol{\%})\end{array}$ & $\mathbf{R}$ & $\mathbf{K}$ & $\mathbf{L S}$ & $\mathbf{C}$ & $\mathbf{P}$ & $\mathbf{A}$ & $\mathbf{E P}$ \\
\hline UEc1 & SP & 60 & 306 & 0,100 & 2,36 & 0,200825 & 1 & 14,5 & 72,22 \\
\hline
\end{tabular}

\footnotetext{
${ }^{6}$ Los planteos productivos seleccionados para la obtención del factor C, corresponden a los mayormente empleados dentro del partido de Benito Juárez en las diversas campañas.
} 


\begin{tabular}{|c|c|c|c|c|c|c|c|c|c|}
\hline & $\mathrm{Az}$ & 40 & 306 & 0,224 & 0,47 & 0,200825 & 1 & 6,5 & 32,21 \\
\hline & & & & & & & & 11,3 & 56,21 \\
\hline \multirow{4}{*}{ UEc2 } & $\mathrm{Ta}$ & 60 & 312 & 0,274 & 0,47 & 0,200825 & 1 & 8,1 & 40,18 \\
\hline & $\mathrm{Az}$ & 30 & 312 & 0,224 & 0,47 & 0,200825 & 1 & 6,6 & 32,85 \\
\hline & $\mathrm{CC}$ & 10 & 312 & 0,176 & 1,86 & 0,200825 & 1 & 20,5 & 102,13 \\
\hline & & & & & & & & 8,89 & 44,17 \\
\hline \multirow{4}{*}{ UEc3 } & Ola & 40 & 312 & 0,252 & 0,47 & 0,200825 & 1 & 7,4 & 36,95 \\
\hline & LLo & 40 & 312 & 0,247 & 0,47 & 0,200825 & 1 & 7,3 & 36,22 \\
\hline & $\mathrm{Az}$ & 20 & 312 & 0,224 & 0,47 & 0,200825 & 1 & 6,6 & 32,85 \\
\hline & & & & & & & & 7,2 & 35,83 \\
\hline \multirow{4}{*}{ UEc4 } & TA & 50 & 318 & 0,202 & 0,42 & 0,200825 & 1 & 5,4 & 26,98 \\
\hline & $\mathrm{LHe}$ & 40 & 318 & 0,488 & 0,1 & 0,200825 & 1 & 3,1 & 15,52 \\
\hline & SIs & 10 & 318 & 0,380 & 0,06 & 0,200825 & 1 & 1,5 & 7,25 \\
\hline & & & & & & & & 4,09 & 20,42 \\
\hline \multirow{5}{*}{ UEc5 } & $\mathrm{LHe}$ & 40 & 318 & 0,488 & 0,1 & 0,200825 & 1 & 3,1 & 15,52 \\
\hline & Ele & 20 & 318 & 0,546 & 0,1 & 0,200825 & 1 & 3,5 & 17,36 \\
\hline & $\mathrm{CBu}$ & 20 & 318 & 0,484 & 0,1 & 0,200825 & 1 & 3.1 & 15,39 \\
\hline & Cru & 20 & 318 & 0,466 & 0,19 & 0,200825 & 1 & 5.7 & 28,15 \\
\hline & & & & & & & & 3,7 & 18,44 \\
\hline
\end{tabular}

Fuente: Elaboración personal a partir de las ecuaciones propuestas por Wischmeier y Smith (1978) y la aplicación web de Gvozdenovich et al. (2015).

TABLA 5

Tasas de erosión actual (A) y potencial (EP) para las campañas 2002/03 y 2014/15

\begin{tabular}{|c|c|c|c|c|c|c|c|c|c|}
\hline Unidad & Serie & $\begin{array}{c}\text { Proporción } \\
(\%)\end{array}$ & $\mathbf{R}$ & $\mathbf{K}$ & $\mathbf{L S}$ & $\mathbf{C}$ & $\mathbf{P}$ & $\mathbf{A}$ & EP \\
\hline \multirow{3}{*}{ UEc1 } & SP & 60 & 306 & 0,100 & 2,36 & 0,06965 & 1 & 5,0 & 72,22 \\
\hline & $\mathrm{Az}$ & 40 & 306 & 0,224 & 0,47 & 0,06965 & 1 & 2,3 & 32,21 \\
\hline & & & & & & & & 3,92 & 56,21 \\
\hline \multirow{4}{*}{ UEc2 } & $\mathrm{Ta}$ & 60 & 312 & 0,274 & 0,47 & 0,06965 & 1 & 2,8 & 40,18 \\
\hline & $\mathrm{Az}$ & 30 & 312 & 0,224 & 0,47 & 0,06965 & 1 & 2,3 & 32,85 \\
\hline & $\mathrm{CC}$ & 10 & 312 & 0,176 & 1,86 & 0,06965 & 1 & 7,1 & 102,13 \\
\hline & & & & & & & & 3,08 & 44,17 \\
\hline \multirow{4}{*}{ UEc3 } & Ola & 40 & 312 & 0,252 & 0,47 & 0,06965 & 1 & 2,6 & 36,95 \\
\hline & LLo & 40 & 312 & 0,247 & 0,47 & 0,06965 & 1 & 2.5 & 36,22 \\
\hline & $\mathrm{Az}$ & 20 & 312 & 0,224 & 0,47 & 0,06965 & 1 & 2.3 & 32,85 \\
\hline & & & & & & & & 2,5 & 35,83 \\
\hline \multirow{4}{*}{ UEc4 } & TA & 50 & 318 & 0,202 & 0,42 & 0,06965 & 1 & 1,9 & 26,98 \\
\hline & $\mathrm{LHe}$ & 40 & 318 & 0,488 & 0,1 & 0,06965 & 1 & 1,1 & 15,52 \\
\hline & SIs & 10 & 318 & 0,380 & 0,06 & 0,06965 & 1 & 0,5 & 7,25 \\
\hline & & & & & & & & 1,44 & 20,42 \\
\hline \multirow{5}{*}{ UEc5 } & $\mathrm{LHe}$ & 40 & 318 & 0,488 & 0,1 & 0,06965 & 1 & 1,1 & 15,52 \\
\hline & Ele & 20 & 318 & 0,546 & 0,1 & 0,06965 & 1 & 1,2 & 17,36 \\
\hline & $\mathrm{CBu}$ & 20 & 318 & 0,484 & 0,1 & 0,06965 & 1 & 1,1 & 15,39 \\
\hline & Cru & 20 & 318 & 0,466 & 0,19 & 0,06965 & 1 & 2,0 & 28,15 \\
\hline & & & & & & & & 1,3 & 18,44 \\
\hline
\end{tabular}

Fuente: Elaboración personal a partir de las ecuaciones propuestas por Wischmeier y Smith (1978) y la aplicación web de Gvozdenovich et al. (2015).

Una vez analizados los resultados, es posible afirmar que poseen cierta similitud con aquellos planteados por Gaitán et al. (2017) para la provincia de Buenos Aires; la cual alcanza tasas medias de A y EP de 2,95 tn ha $^{-1}$ año $^{-1}$ y 36,56 tn ha $^{-1}$ año $^{-1}$, respectivamente .

\footnotetext{
${ }^{7}$ Los datos pertenecientes al citado trabajo se encuentran calculados considerando la práctica de Siembra Directa. Por lo tanto, debido a que en la campaña 1990/91 en el partido de Benito Juárez la agricultura se
} 
Además, resulta factible establecer diferencias notables entre los valores de A alcanzados en las diversas UEcs. Para la campaña 1989/90, el valor más elevado se obtuvo para la UE1 (11,3 t $\left.\mathrm{ha}^{-1} \mathrm{año}^{-1}\right)$ y el más bajo en la UEc5 $\left(3,7 \mathrm{t} \mathrm{ha}^{-1} \mathrm{año}^{-1}\right)$. Por otra parte, se evidencia que para las campañas 2002/2003 y 2014/2015 la tendencia se repitió, volviendo a destacarse la UEc1 con el valor más elevado $\left(3,92 \mathrm{t} \mathrm{ha^{-1 }}\right.$ año $\left.{ }^{-1}\right)$ y la UEc5 con el más bajo $\left(1,3 \mathrm{t} \mathrm{ha}{ }^{-1}\right.$ año $\left.^{-1}\right)$.

En relación a la EP, se obtuvieron los mismos valores para las 3 campañas. La explicación de este escenario radica en que, al ser considerada como la máxima posible para un determinado sitio (teniendo en cuenta un suelo desnudo todo el año,) los valores de los factores relacionados a la cobertura y las prácticas conservacionistas no poseen influencia sobre el resultado. Sumado a eso, y considerando la relación existente entre EP y A, nuevamente se destacan las mismas UEcs, siendo el valor más elevado el registrado en la UEc1 y el más bajo en la UEc5, con 56,21 t ha-1 año ${ }^{-1}$ y $18,44 \mathrm{t} \mathrm{ha}^{-1} \mathrm{año}^{-1}$, respectivamente.

\subsection{PÉRDIDA TOTAL DE SUELOS POR EROSIÓN HÍDRICA}

Posteriormente a la obtención de los resultados de A y EP por UEc, y contando con la superficie agrícola de cada UEc por año analizado (datos alcanzados gracias a la superposición de las UEcs y las CS), fue posible realizar una estimación acerca de la pérdida total de suelos por erosión hídrica en cada una de ellas. Para llevar esto a cabo, los valores de A y EP fueron multiplicados por la superficie dedicada a la actividad agrícola en cada UEc, obteniéndose de esta manera la pérdida expresada en tn año ${ }^{-1}$ (Tabla 6).

TABLA 6

Pérdida total de suelos para las campañas 1989/90, 2002/03 y 2014/15

\begin{tabular}{|c|c|c|c|}
\hline Unidad & $\begin{array}{c}\text { Sup. agrícola } \\
\text { (ha) }\end{array}$ & $\begin{array}{c}A \\
\left(\operatorname{tn} a_{\tilde{n}}^{-1}\right)\end{array}$ & $\begin{array}{c}\mathbf{E P} \\
\left(\operatorname{tn} \mathbf{a n ̃ o}{ }^{-1}\right)\end{array}$ \\
\hline \multicolumn{4}{|c|}{ Campaña 1989/90 } \\
\hline UEc1 & 1370 & 15481 & 77007.7 \\
\hline UEc2 & 12018 & 106840,02 & 530835,06 \\
\hline UEc3 & 22811 & 164239,2 & 817318,13 \\
\hline UEc4 & 5206 & 21292,54 & 106306,52 \\
\hline UEc5 & 20578 & 76138,6 & 379458,32 \\
\hline \multicolumn{4}{|c|}{ Campaña 2003/03 } \\
\hline UEc1 & 2448 & 9596,16 & 137602,08 \\
\hline UEc2 & 23424 & 72145,92 & 1034638,08 \\
\hline UEc3 & 47804 & 119510 & 1712817,32 \\
\hline UEc4 & 7201 & 10369,44 & 147044,42 \\
\hline UEc5 & 50057 & 65074,1 & 923051,08 \\
\hline \multicolumn{4}{|c|}{ Campaña 2014/15 } \\
\hline UEc1 & 3038 & 11908,96 & 170765,98 \\
\hline UEc2 & 42233 & 130077,64 & 1865431,61 \\
\hline UEc3 & 113840 & 284600 & 4078887,2 \\
\hline UEc4 & 18285 & 26330,4 & 373379,7 \\
\hline UEc5 & 116920 & 151996 & 2156004,8 \\
\hline
\end{tabular}

Fuente: Elaboración personal.

Al analizar los datos, se observa que en el período 1989/90-2002/03 los valores de A demuestran un claro decrecimiento. Sin embargo, en el período 2002/03-2014/15 la situación fue inversa, y queda demostrado que los valores alcanzados sufrieron un incremento. Por su parte, los valores de EP evidencian un notable crecimiento en ambos períodos.

Prosiguiendo con el análisis es factible plantear que, para las 3 campañas, la mayor pérdida total de suelos ocurrió dentro de la UEc3. En ella, para el primer período, el valor de A evidenció

desarrollaba bajo labranza tradicional, solo pudieron ser comparados con los valores de A y EP de las campañas 2002/03 y 2014/15. 
un decrecimiento del 27,23\%, y el de EP creció en un 109,56\%. Para el segundo período, tanto el resultado de A como así también el de EP se elevaron en un 138,14\%.

\subsection{TOLERANCIA A LA PÉRDIDA DE SUELO}

Una vez obtenidos los valores de la tasa de erosión actual (A) y de tolerancia a la pérdida de suelos (Tol) de cada UEc, es posible contrastarlos y definir si el sistema productivo es sostenible o no, desde el punto de vista de la erosión hídrica (Tabla 7). Para ello se establece que, si el valor de A es mayor al de Tol, el sistema genera erosión y no es sostenible. Contrariamente, si resulta menor, se considera que no genera erosión y por lo tanto es sostenible (IRURTIA y CRUZATE, 2002).

TABLA 7

Tasas de A y Tol por UEc para las campañas 1989/90, 2002/03 y 2014/15

\begin{tabular}{|c|c|c|c|}
\hline Unidad & $\begin{array}{c}\text { Erosión actual } \\
\left(\text { tn ha }^{-1} \text { año }^{-1}\right) \\
1989 / 1990\end{array}$ & $\begin{array}{c}\text { Erosión actual } \\
\left(\text { tn ha }^{-1} \text { año }^{-1}\right) \\
2002 / 03-2014 / 15\end{array}$ & $\begin{array}{c}\text { Tolerancia } \\
\left(\text { tn ha }^{-1} \mathbf{a n ̃ o}^{-1}\right)\end{array}$ \\
\hline UEc1 & 11,3 & 3,92 & 3,75 \\
\hline UEc2 & 8,89 & 3,08 & 5,5 \\
\hline UEc3 & 7,2 & 2,5 & 5,5 \\
\hline UEc4 & 4,09 & 1,44 & 5,5 \\
\hline UEc5 & 3,7 & 1,3 & 5,125 \\
\hline
\end{tabular}

Fuente: Elaboración personal.

Para la campaña 1989/90, las UEc1, UEc2 y UEc3 presentan valores de A superiores al límite de Tol, por lo que pueden clasificarse como no sostenibles. En contraposición, las UEc4 y UEc5 resultan sostenibles, al demostrar valores de A por debajo de los de Tol.

En las campañas 2002/03-2014/15, solamente la UEc1 resulta no sostenible; mientras que el resto presenta valores de A inferiores. Esta variación en los datos alcanzados puede explicarse, nuevamente, al considerar que a partir del año 2002 comenzó a implementarse la SD.

Luego de adquiridos los resultados, puede establecerse una correlación con lo sucedido a nivel país, donde casi el 25,9\% del territorio nacional (alrededor de 72 millones de hectáreas) presenta tasas de erosión hídrica que superan los valores tolerables (GAITÁN et al., 2017).

\subsection{RELACIÓN ENTRE VALORES DE EROSIÓN ACTUAL Y POTENCIAL, PÉRDIDA TOTAL DE SUELOS Y AGRICULTURIZACIÓN}

Como ya fue mencionado, la agricultura se encuentra entre las principales actividades responsables de la erosión del suelo. Además, sus efectos negativos dependen directamente de las características propias de la zona sobre la cual se lleva a cabo la actividad, y de los tipos de prácticas efectuados.

Una vez analizada la totalidad de los datos obtenidos acerca de la variación de los valores de A y EP, y de pérdida total de suelos en las distintas UEcs que constituyen al partido de Benito Juárez; es posible plantear que existe una relación directa entre los resultados alcanzados y el incremento de la superficie destinada al desarrollo de la agricultura.

En primer lugar, se puede observar que entre 1989/90 y 2002/03 creció la superficie agrícola en las 5 UEcs. Pero, al mismo tiempo, los valores de A disminuyeron en todas ellas. Lo sucedido puede ser explicado, en parte, debido a la variación del método de labranza utilizado dentro del Partido, el cual pasó de SC a SD.

Sin embargo, en el período 2002/03-2014/15 la situación fue inversa. Aunque se siguió utilizando SD, la pérdida total de suelos se incrementó como consecuencia de, principalmente, un nuevo aumento de la superficie destinada a la agricultura. Este escenario permite establecer que la modificación del tipo de siembra, no resulta suficiente para disminuir los efectos negativos de la actividad sobre el suelo. 
Lo indicado concuerda con planteos de varios autores. Según José Luis Panigatti (ex presidente de la Asociación Argentina de Ciencias del Suelo), la SD no es por sí misma una solución definitiva. Se trata de una práctica que debe ser complementada con un adecuado plan de rotaciones de cultivos, una fertilización apropiada y con la decisión de otorgarle al suelo la mayor combinación de elementos que permitan conservarlo (CASTILLA, 2013).

Sumado a lo anterior, Miguel Taboada (representante argentino en la Alianza Mundial del Suelo) afirma que la mencionada rotación de cultivos resulta ser clave, debido a que mejora el balance de los nutrientes y la materia orgánica en los suelos, el aprovechamiento del agua y posee un efecto inhibitorio sobre diversas plagas, malezas y enfermedades (CASTILLA, 2013), logrando alcanzar así una mejor gestión del suelo como recurso.

\section{CONCLUSIONES}

La apreciación y análisis de los impactos negativos del proceso de agriculturización, comienzan a tener cada vez mayor relevancia dentro del ámbito académico. Entre las herramientas destinadas a tal fin, la Ecuación Universal de Predicción de Erosión Hídrica conforma un claro indicador para estimar las tasas de erosión en aquellas áreas donde se desarrollan actividades agrícolas.

Para el presente trabajo, la ecuación fue aplicada a las distintas UEcs que conforman al partido de Benito Juárez, en 3 campañas agrícolas. Los resultados demuestran que los valores de A y EP, y la pérdida total de suelos presentan variaciones. Este escenario puede explicarse, en cierta medida, por las características propias de las UEcs, y por las diferencias en relación a la superficie dedicada a la agricultura en cada una de ellas.

El hecho de concretar una estimación de la variación temporal de A y de la pérdida de suelos, resulta de gran relevancia al momento de efectuar un diagnóstico sobre la situación del modelo productivo del Partido, analizando en qué medida este resulta realmente sostenible.

Aunque los valores de A y su relación con la tolerancia a la pérdida de suelos demuestran ciertas mejoras (relacionadas principalmente a la aplicación de SD), debería concretarse una investigación más profunda. Si bien se encuentra por debajo de lo tolerable, la erosión continúa siendo un grave impacto negativo.

En relación a ello se establece la necesidad de, en próximos trabajos, efectuar nuevas evaluaciones que consideren una mayor diversidad de secuencias de cultivos (factor C), estimando su potencial pérdida de suelos; $\mathrm{y}$, de esta manera, plantear rotaciones tendientes a una reducción de las pérdidas por erosión.

Finalmente, se considera que la metodología aplicada permite recabar información útil al momento de establecer un correcto ordenamiento ambiental del territorio respecto al uso de los suelos agrícolas; y, además, puede ser replicada en otras áreas de interés, con el propósito de evaluar posibles medidas de mitigación en aquellas regiones afectadas por elevadas tasas de erosión.

\section{BIBLIOGRAFÍA}

ALTIERI, M. (1999): Agricultura tradicional y la conservación de la biodiversidad. "Biodiversidad y uso de la tierra. Conceptos y ejemplos de Latinoamérica". En Matteucci, S., Solbrig, O., Morello, J. y Halffter, G. (Eds.). EUDEBA. Buenos Aires, pp. 71-83.

ARES, M. G., VARNI, M. R. y CHAGAS, C. I. (2014): "Erosión hídrica en una microcuenca agrícola bajo siembra directa del centro de la provincia de Buenos Aires". Ciencia del Suelo, vol. $32, n^{\circ} 2$, p. 259-270.

BILENCA, D., CODESIDO, M., FISCHER, C. G., CARUSI, L. P., ZUFIAURRE, E. y ABBA, A. (2012): "Impactos de la transformación agropecuaria sobre la biodiversidad en la provincia de Buenos Aires". Revista del Museo Argentino de Ciencias Naturales, vol. 14, nº 2, p. 189198.

BONILLA, C. A., REYES, J. L. y MAGRI, A. (2010): "Water erosion prediction using the Revised Universal Soil Loss Equation (RUSLE) in a GIS framework, central Chile". Chilean Journal of Agricultural Research, vol. 70, $\mathrm{n}^{\circ}$ 1, p. 159-169. 
CABRERA, A. L. (1976): Regiones Fitogeográficas Argentinas. Enciclopedia Argentina de Agricultura y Jardinería. Buenos Aires: Editorial: ACME (TOMO II.).

CASAS, R. (2015): Degradación de Tierras en la República Argentina. "La erosión del suelo en la Argentina". En Casas, R. y Albarracín, G. (Eds.). FECIC. Buenos Aires, pp 433- 452.

CASTILLA, F. (2013): "Siembra directa. La elegida para conservar el suelo". RIA. Revista de investigaciones agropecuarias, vol. 39, $\mathrm{n}^{\circ}$ 2, p. 118-123.

CERDÁ, E; SARANDÓN, S. y FLORES, C. (2014): Agroecología: bases teóricas para el diseño y manejo de Agroecosistemas sustentables. "El caso de "La Aurora": un ejemplo de aplicación del enfoque agroecológico en sistemas extensivos del sudeste de la provincia de Buenos Aires, Benito Juárez, Argentina". En Sarandón, S. y Flores, C. (Eds.). Servicio de publicaciones, Universidad Nacional de La Plata. La Plata, pp. 437-463.

FOOD AND AGRICULTURE ORGANIZATION OF THE UNITED NATIONS (FAO). (1980): Metodología provisional para la evaluación de la degradación de los suelos. Organización de las Naciones Unidas para la Agricultura y Alimentación. Roma. 85 pp.

FOOD AND AGRICULTURE ORGANIZATION OF THE UNITED NATIONS (FAO). (1990): Guidelines for soil description. Rome, Italy.

FERRERAS, L. A., TORESANI, S. M. I., FAGGIOLI, V. S. y GALARZA, C. M. (2015): "Sensibilidad de indicadores biológicos edáficos en un Argiudol de la Región Pampeana Argentina". Sociedad Española de la Ciencia del Suelo, vol. 5, nº 3, p. 227-242.

GAITÁN, J., NAVARRO, M. F., VUEGEN, L. T., PIZARRO, M. J., CARFAGNO, P. y RIGO, S. (2017): Estimación de la pérdida de suelo por erosión hídrica en la República Argentina. Colección: Investigación, desarrollo e innovación INTA. Ediciones INTA, 66 pp.

GARCÍA, F., y SALVAGIOTTI, F. (2009): Eficiencia de uso de nutrientes en sistemas agrícolas del Cono Sur de Latinoamérica. XVIII Congreso Latinoamericano de la Ciencia del Suelo, San José, Costa Rica, 16-20 de noviembre. San José, Instituto Interamericano de Cooperación para la Agricultura (IICA), pp. 37-48.

GONZÁLEZ, M. D. C. y ROMÁN, M. (2009): "Expansión agrícola en áreas extrapampeanas de la Argentina. Una mirada desde los actores sociales". Cuadernos de Desarrollo Rural, vol. 6, $\mathrm{n}^{\circ} 62$, p. 99-120.

GVOZDENOVICH, J.; P. BARBAGELATA y G. LÓPEZ (2015): Erosión Hídrica USLE/RUSLE Argentina-INTA EEA Paraná. Software, Versión 2.0. URL: http://www.inta.gob.ar/parana

GVOZDENOVICH, J., PÉREZ BIDEGAIN, M., NOVELLI, L. y BARBAGELATA, P. (2017): “¿Puede WEPP mejorar la predicción de la erosión de suelos respecto a USLE?”. Ciencia del suelo, vol. $35, \mathrm{n}^{\circ} 2$, p. 259-272.

HERNÁNDEZ, L., y DUFILHO, A. C. (2012): "Determinación del riesgo de erosión hídrica de los suelos de los departamentos Minas y Chos Malal (Neuquén) mediante la aplicación de la ecuación universal de pérdida de suelo (USLE)". Boletín geográfico, ${ }^{\circ} 34$, p. 11-31.

IRURTIA, C. y CRUZATE, G. (2002): Aplicación de la Ecuación Universal de Pérdida de Suelo en la Provincia de Buenos Aires. Informe Técnico. INTA-Castelar, 14 pp.

LÓPEZ, F. M., DUVAL, M. E., MARTÍNEZ, J. M. y GALANTINI, J. A. (2015): "Cobertura en el sudoeste bonaerense en suelos bajo siembra directa". Ciencia del suelo, vol. 33, n 2, p. 273 281.

MANCHADO, J. (2010): La sustentabilidad en la agricultura pampeana: Valoración económica del balance de nutrientes para las principales actividades agropecuarias extensivas en la Región Centro Sur de la Provincia de Buenos Aires. XLI Reunión anual de la Asociación Argentina de Economía Agraria. Potrero de los Funes, San Luis, Argentina, 6-8 de octubre. Argentina, Asociación Argentina de Economía Agraria (AAEA), pp. 1-18.

MARTÍNEZ, A. N. e IGLESIAS, A. N. (2006): Elementos Jurídicos-Normativos de la ordenación ambiental del territorio en Argentina. Significado de la escala local de gestión. https://estrucplan.com.ar/articulos/verarticulo.asp?IDArticulo=1253 [consulta: marzo 20, 2018].

NÁJERA GONZÁLEZ, O., BOJÓRQUEZ SERRANO, J. I., FLORES VILCHEZ, F., MURRAY NÚÑEZ, R. M. y GONZÁLEZ GARCÍA-SANCHO, A. (2016): "Riesgo de erosión hídrica y 
estimación de pérdida de suelo en paisajes geomorfológicos volcánicos en México". Cultivos Tropicales, vol. $37, \mathrm{n}^{\mathrm{o}} 2$, p. 45-55.

NOCELLI PAC, S. (2018): Evolución de siembra directa en Argentina, campaña 2016-2017. Informe elaborado para Aapresid (Asociación Argentina de Productores en Siembra Directa), $8 \mathrm{pp}$.

FECIC (Fundación para la Educación, la Ciencia y la Cultura)-PROSA (Centro para la Promoción de la Conservación del Suelo y del Agua). (1988): El Deterioro del Ambiente en la Argentina. FECIC. Editorial Orientación Grafica Editora.

DÍAZ PEREIRA, E. y ANAYA ROMERO, M. (2011): "Modelos de evaluación agroecológica de tierras: erosión y contaminación en el entorno MicroLEIS". Teoría y Praxis, n 9, p. 91107.

REQUESENS, E. y SILVA, L. (2011): “Tendencias en el uso de la tierra y diversidad productiva en establecimientos agropecuarios del centro-sur de la provincia de Buenos Aires (Argentina)". Agriscientia, vol. 28, $\mathrm{n}^{\mathrm{o}}$ 1, p. 75-83.

SALINAS CHÁVEZ, E. y RAMÓN PUEBLA, A. (2013): "Propuesta metodológica para la delimitación semiautomatizada de unidades de paisaje de nivel local". Revista do departamento de geografia, vol. 25, p. 1-19.

SANS, F. (2007): "La diversidad de los agroecosistemas". Revista ecosistemas, vol. 16, $\mathrm{n}^{\mathrm{o}}$ 1, p. 44-49.

SERRANO GINÉ, D.S. (2012): "Consideraciones en torno al concepto de unidad de paisaje y sistematización de propuestas". Estudios geográficos, vol. 73, nº 272, p. 215-237

SEQUEIRA, N.; VAZQUEZ, P. y ZULAICA, L. (2015): "Consecuencias ambientales de la expansión agrícola en el partido de Benito Juárez (Buenos Aires, Argentina), en el período 2003-2011". Revista Geoaraguaia, vol. 5, n 2, p. $26-49$.

SEQUEIRA, N. D., VAZQUEZ, P. y SACIDO, M. (2018): Expansión agrícola y riesgo de contaminación por plaguicidas, el caso del partido de Benito Juárez. II Jornadas Internacionales y IV Jornadas Nacionales de Ambiente. Tandil, Buenos Aires, Argentina, 1719 de octubre. Facultad de Ciencias Humanas, Universidad Nacional del Centro de la Provincia de Buenos Aires, Tandil, pp. 445-448.

SEQUEIRA, N. D., VAZQUEZ, P. y ZULAICA, L. (2019): "Definición y caracterización de Unidades Ecológicas en el partido de Benito Juárez (Argentina): aportes para planificar el uso sustentable de los recursos". Revista Ciencias Agronómicas, vol. 33, n 19, p. 031-038.

SOCIEDAD RURAL DE JUÁREZ (2007): Evaluación de tierras del Partido de Benito Juárez. Estudio técnico contratado al Laboratorio de Geomática de la Unidad Integrada Balcarce INTA-Facultad de Ciencias Agrarias (UNMP).

VAZQUEZ, P., SACIDO, M., y ZULAICA, L. (2013): “Zonificación agroecológica de la cuenca del río Quequén Grande (provincia de Buenos Aires, Argentina)". Geoaraguaia, vol. 3, nº 2, p. $26-45$ p.

WISCHMEIER, W. H. y SMITH, D. D. (1978): Predicting rainfall erosion losses - a guide to conservation planning. Maryland, USA: Science and Education Administration, USDA, pp. 62.

ZULAICA, L.; VAZQUEZ, P. y DAGA, D. (2018): Estimación de la erosión actual y potencial de suelos destinados a actividades hortícolas en el periurbano de Mar del Plata. V Jornadas Nacionales de Investigación en Geografía Argentina y XI Jornadas de Investigación y Extensión del Centro de Investigaciones Geográficas. Tandil, Buenos Aires, Argentina, 16-19 de mayo. Facultad de Ciencias Humanas, Universidad Nacional del Centro de la Provincia de Buenos Aires, Tandil, pp. 805-817. 